\title{
Commentary: What are behavior systems and what use are they?
}

\author{
SARA J. SHETTLEWORTH \\ University of Toronto, Toronto, Ontario, Canada
}

\begin{abstract}
The contributions of this symposium on behavior systems are summarized and evaluated by considering two questions: (1) What is a behavior system? (2) What use to the learning theorist are behavior systems? Two examples of behavior systems from the classical ethological literature are compared with the behavior systems discussed in the symposium, and some similarities and differences in the type of analyses used are discussed. Analysis of the preorganized species-typical behavior systems relevant to the unconditioned stimuli or reinforcers in learning experiments can contribute performance rules and better understanding of the conditions and contents of learning. The organization of behavior systems can also provide important clues to the neural circuitry underlying behavior, and a behavior systems approach can raise novel questions concerning learning and behavioral development. Possible future directions for the behavior systems approach are briefly discussed.
\end{abstract}

The contributors to this symposium deserve our thanks for reviewing in one place four successful and interesting research programs. Although these programs are diverse in the questions they ask and the methods they use, each of them has made extensive use of the notion that behavior is organized hierarchically into motivational systems. In his introduction, Timberlake (1994) claims that this approach can unify the disparate contemporary approaches to learning. Here, I briefly address Timberlake's claim by discussing two questions. First, what is a behavior system? Second, what use to the learning theorist are behavior systems?

\section{What Is a Behavior System?}

Readers may be struck by the fact that each contribution to the symposium contains some sort of diagrammatic representation of a behavior system: fear in the rat (Fanselow, 1994), feeding in the rat (Timberlake, 1994), courtship in quail (Domjan, 1994), and dustbathing and feeding in chickens (Hogan, 1994). One thing these have in common is their hierarchical organization. Implicitly or explicitly, the lowest level of the hierarchy is a set of species-typical motor patterns. As both Hogan and Timberlake point out, the idea that species-typical behavior is organized into motivational systems of this general nature is at the core of classical ethology. Comparing the schemes for behavior systems presented here with some older ethological examples reveals some important similarities and differences and raises a few questions.

Preparation of this commentary was supported by a research grant to the author from the Natural Sciences and Engineering Research Council of Canada. I thank Bill Timberlake for inviting me to participate in the symposium and Jerry Hogan for commenting on a draft of the manuscript. Correspondence should be addressed to $\mathrm{S}$. J. Shettleworth, Department of Psychology, University of Toronto, Toronto, ON, Canada M5S 1A1 (e-mail: shettle@psych.toronto.edu).
Consider, for example, nest provisioning behavior of the digger wasp, Ammophila adriaansei (Baerends, 1976), depicted in Figure 1. The wasp lays eggs in a number of underground burrows and provisions each of them with caterpillars to feed the larvae when they hatch. At any one time, she may have several nests in various stages of completion. When she visits an uncompleted nest, the amount of food still available for the larva as well as the larva's size determine (via the decision unit labeled HS [heterogeneous summation] in Figure 1) whether she will hunt for new caterpillars to bring to that nest, inspect another nest, or start a new one. This reproductive behavior ( $R p$ in Figure 1$)$ is mingled with maintenance behavior $(\mathrm{Mt})$, such as feeding, grooming, and sunbathing.

At first glance, the layout of Figure 1 appears parallel to that of Timberlake's (1994) depiction of the rat's feeding system. The lowest (rightmost) level in each behavioral hierarchy consists of species-typical motor patterns, such as grasping, siezing, and munching prey. These are organized into groups named according to their function-digging, investigating, closing, and the like-or modules in Timberlake's terminology. These groups of behavior are organized into larger functional groups (modes, subsystems): provisioning, general search, and so on.

So far, we might summarize this kind of picture (which resembles in varying degrees that presented elsewhere in the symposium) by saying that behavior consists of groups of well-defined motor patterns that share a common function. These functional groups of motor patterns will also tend to occur together in time, partly because motor patterns that require the same stimulus support and depend on the same internal state tend to be grouped together. For instance, seizing, stinging, and munching (components of the hunting module in Figure 1) require a caterpillar prey item, whereas raking 
and excavating (digging) require sand. All these activities occur only when the wasp is in reproductive condition. This aspect of behavior systems is part of what is captured by Hogan's (1994) definition of a behavior system as "perceptual, central, and motor elements that function as a unit in some situations." A closer comparison of Timberlake's (1994, Figure 1) scheme for feeding in the rat with Baerends's depiction of the wasp's provisioning behavior shows that these perceptual elements, as well as the interactions among central elements, are more fully represented in Baerends's scheme. For example, the upper left corner of Figure 1 is a representation of the wasp's memory for the current uncompleted nests (a-e) and the focus of its attention on one at a time (heavy lines) until each provisioning phase is completed. Thus, in addition to indicating how behaviors are grouped together functionally, a diagram such as Figure 1 indicates, at least roughly, the relevant stimulus input and the control mechanisms that cause the animal to change from doing one thing to doing another. Some of this sort of information is incorporated in Timberlake's (1994) Figures $2 a-2 c$, but, it seems to me, at the expense of almost seeming to limit the behavior under consideration to a chain of reflexes.

Figure 2 shows that the workings of a behavior system can be depicted in considerable detail. Indeed, Figure 2 might be the sketch of a wiring diagram for a gull robot (see McFarland \& Bösser, 1993). To a much greater degree than Figure 1, this diagram of interruptive behavior during incubation by herring gulls incorporates the influence of external stimuli (note the frightening stimulus in the upper right, the eggs, and the dust and parasites stimulating preening in the lower right), decision mechanisms (top of the figure), and excitatory and inhibitory interactions among subsystems. It takes almost to its limit the kind of scheme for the organization of behavior laid out in Hogan's (1994) Figure 1.

I have two reasons for presenting these examples. First, although the ethological concept of behavior system is mentioned elsewhere in this symposium, Figures 1 and 2 make clear how closely related to it is the present research discussed here. These examples, from an insect and a bird and from behaviors very different from those discussed in other contributions to the symposium, also support any claims in the other articles about the general

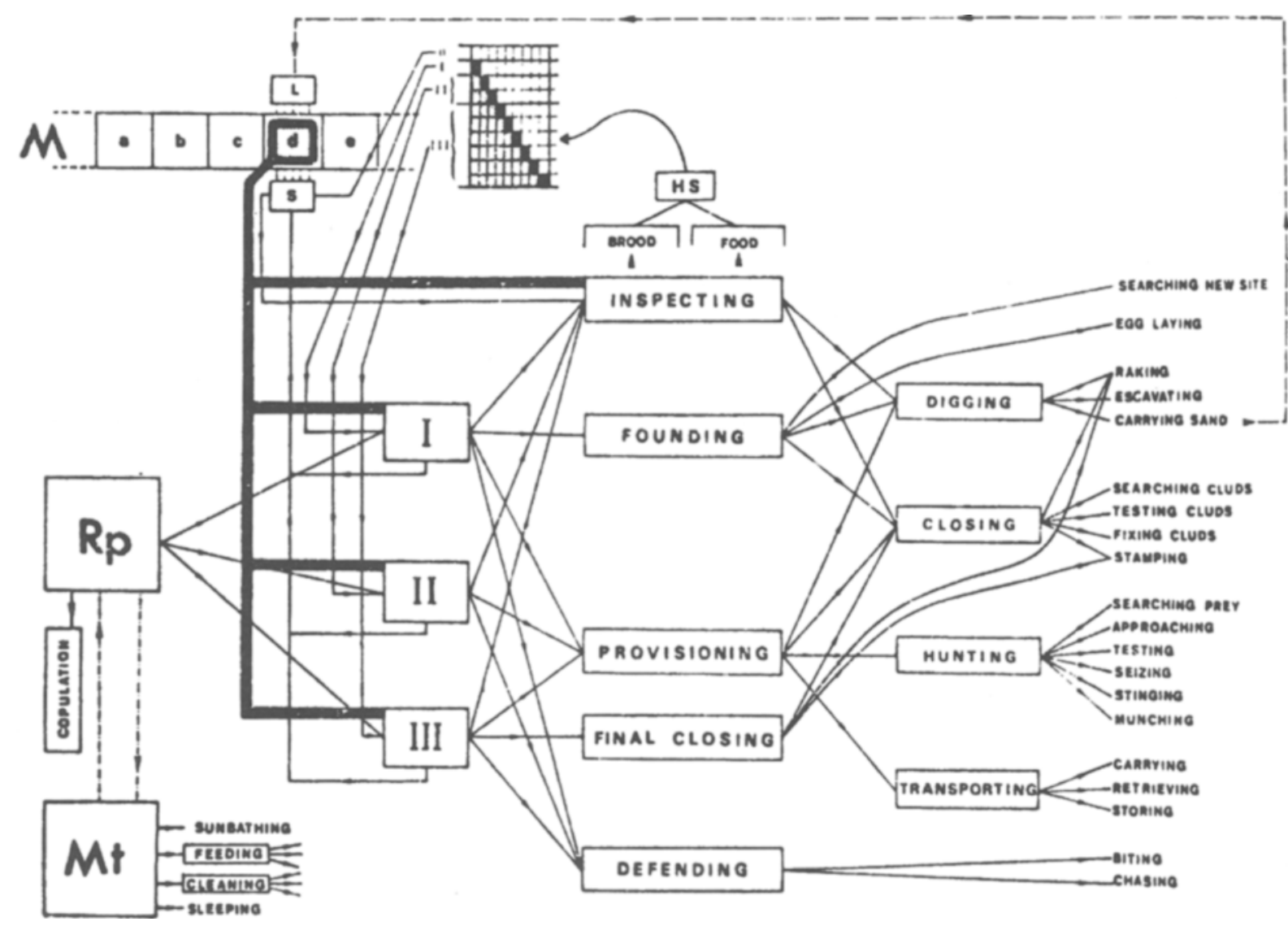

Figure 1. Hierarchical organization of nest provisioning behavior in the digger wasp, Ammophila adriaanseii. The motor patterns involved are listed on the right side of the figure, and their organization into functional groups is indicated by lines connecting them to higher levels in the hierarchy. I, II, and III are stages of provisioning behavior. The role of memory for several nests in different stages of completion is indicated in the upper left, as discussed further in the text. Note--From "The Functional Organization of Behavior" by G. P. Baerends, 1976, $A n$ imal Behaviour, 24, p. 728. Copyright 1976 by Academic Press. Reprinted by permission. 


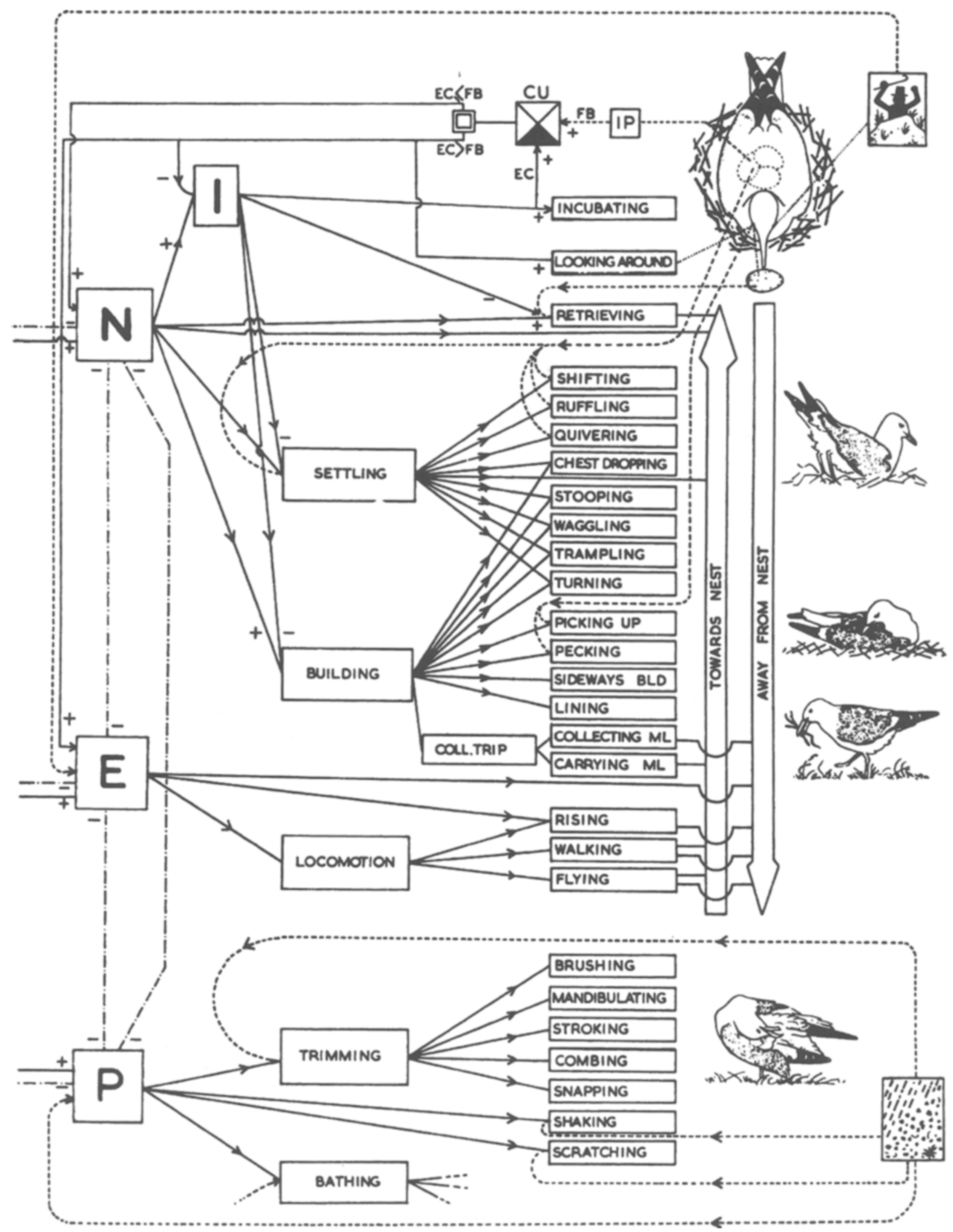

Figure 2. Organization of interruptive behavior such as preening and leaving the nest during incubation by the herring gull. The behaviors involved are depicted as belonging to three interacting systems: incubation $(N)$, escape $(E)$, and preening (P). When feedback from the eggs matches expectancy, incubation is facilitated and the various interruptive behaviors are inhibited (I), as depicted at the top of the figure. NoteFrom "The Herring Gull and Its Egg: Part C. A Model of the Functional Organization of Incubation Behavior" by G. P. Baerends, 1970, Behaviour, I7, p. 267. Copyright 1970 by E. J. Brill. Reprinted by permission. 
applicability of the behavior systems approach. Of course, the parallels among the analyses that the four symposium contributors find useful also speak to the power of this general approach to encompass a variety of specific species and behaviors.

My second reason for presenting Figures 1 and 2 is to point out that a behavior systems approach can lead to analyses differing greatly from one another not only in degree of detail (compare them, for example, with the depictions in the contributions by Fanselow and Domjan) but also in kind. This difference in kind is essentially what Hogan refers to as that between structural (or causal) and functional definitions of behavior systems. It is the difference between analyses with an explicit role for the input side of behavior and central decision mechanisms (as in the present Figure 2) and analyses in which behaviors are grouped together apparently by the function they serve with no explicit role for input. I am not suggesting that all, or even any, of the present authors should devote themselves to refining the analysis of their favorite behavior system to the level implicit in Figure 2. In fact, it is clear from the text of each of the articles that the authors already have a detailed understanding of the influence of specific stimuli and central states on their subjects' behavior. They know more than they make explicit about why their animals do what they do. This is shown, for example, in Timberlake's (1994) discussion of rats' behavior toward a moving ball bearing and Fanselow's (1994) discussion of the role of supporting stimuli in the various subsystems of defensive behavior. People working in this area might want to consider whether communication (and perhaps analysis) would be facilitated by formalizing some of this understanding in the style of Baerends (see Figures 1 and 2). It might also be worth considering whether different levels and kinds of analysis are useful for different purposes.

\section{What Use Are Behavior Systems?}

To some, it may seem too obvious to need repeating that behavior, in terms of species-typical motor patterns, is organized hierarchically. The idea has a long history in neuroscience as well as in ethology (see Gallistel, 1980). However, Timberlake's claim in the introduction to the present symposium is that the understanding of learning, and particularly the integration of different approaches to studying learning, will be improved by looking at learning as acting within preorganized behavior systems. Learning, the claim is, changes not just the strength of associations but the behavioral organization of a whole animal.

Investigations of associative learning address three primary issues (Rescorla, 1988): the conditions of learning, the contents of learning, and the effects of learning on behavior. Other important questions in contemporary research include the neural basis of learning, how apparently different kinds of learning differ from one another, and how learning develops and has evolved (Shettleworth, 1993). The contributions to this symposium provide unique insights into all of these issues.
Performance rules. Although the current authors barely mention them, the puzzles created by autoshaping, animal "misbehavior," and "constraints on learning" more than 20 years ago were important stimuli for looking at behavior systems in the context of learning (for review, see Domjan, 1983). All of these phenomena indicated that an animal's preexisting behavioral organization plays a crucial role in determining what it does in standard laboratory learning paradigms. The research described by Fanselow (1994) is the direct continuation of one line of work that began at that time, stimulated by the observation that animals seemed able to perform only certain kinds of responses (species-specific defense reactions; Bolles, 1970) in avoidance situations. The experiments with rats and ball bearings described by Timberlake (this issue) also have their roots in the puzzles of animal misbehavior (Breland \& Breland, 1961). Several of the classic cases of misbehavior involved animals performing species-typical feeding or predatory responses with tokens that had been paired with food reinforcement. Fanselow and Timberlake clearly show that an independent analysis of the behavior system related to a given reinforcer or unconditioned stimulus (US) can account for performance in conditioning situations (see also Shettleworth, 1975). Domjan (1994) discusses how it can account for the influence of the conditioned stimulus (CS) on the nature of the conditioned response (CR) as well.

Learning rules. But Timberlake (1994) claims that a behavior systems approach can predict learning as well as performance. He gives several examples. My favorite is a comparative one: rats, which are social feeders, learn to treat a conspecific as a cue to food, whereas hamsters, which are solitary except for mating, seem indifferent to another hamster that predicts the presence of food. It might be suggested that this particular case could be even more thoroughly analyzed to rule out the possibility that the hamster subjects did actually learn something about the hamster CS. Nevertheless, Timberlake makes a compelling argument that analysis of behavior systems allows predictions about what and under what conditions particular species will learn.

One condition for learning that receives special attention by the symposium contributors is the CS-US interval. Both Timberlake and Domjan show how different parts of a behavior system may be conditioned, depending on the CS-US interval. Handling and consumption are conditioned at the shortest intervals, focal search at slightly longer ones, and general search at the longest intervals. It is noteworthy that this analysis applies equally to feeding in rats (Timberlake, 1994) and courtship in quail (Domjan, 1994). As these authors acknowledge, their analysis has a precedent in, among other things, Konorski's (1967) division of CRs into preparatory and consummatory responses. However, the power of the approach developed here is that it shows how speciestypical preparatory and consummatory responses can be identified independently of the learning situation in question. It also ties the preparatory-consumatory dis- 
tinction to a broader body of theory and data in behavioral science.

Contents of learning. The CS-US interval is generally depicted as affecting the strength of association: the longer the interval, the weaker the association. However, the preceding analysis implies that different things are learned at different CS-US intervals. The data presented by Timberlake and Domjan in this regard are supported by Fanselow's (1994) demonstration that defensive behavior consists of two systems, circa-strike behavior and postencounter behavior, defined by their temporal relation to the aversive event and having different physiological substrates.

Hogan's (1994, Figure 1) general depiction of behavior systems leads to a provocative suggestion about the contents of learning. He suggests that the connections among and within the different elements of behavior systems-perceptual, central, and motor-are acquired in different ways. That is, what is being learned will predict what kind of learning will be involved. For example, classical conditioning involves the formation of perceptual-central connections, whereas instrumental conditioning is the connection of preexisting motor units to central units. Hogan also suggests that his scheme for behavior systems parallels contemporary representational accounts of associative learning. Given the usefulness of the behavior systems approach as demonstrated by all four contributions to the symposium, an attempt to bring together representational and behavior system accounts of learning in more detail could be very worthwhile.

Behavior systems and the brain. In his introduction to the symposium, Timberlake claims that a behavior systems approach can unify diverse approaches to learning, including physiological ones. Fanselow's (this issue) contribution shows in detail how this can be done by mapping observed interactions among behavioral subsystems into systems in the brain. Although much of the analysis of learning at the neural level has focused on systems with a single simple $\mathrm{CR}$, such as an eyeblink, the research program described by Fanselow shows beautifully how more complex systems of learned behaviors can be analyzed physiologically and how vital sophisticated behavioral observations can be to the success of such work.

New questions about learning: How do behavior systems develop? Not only does a behavior systems approach help to answer questions about learning that arise in other approaches, it raises new questions of its own. The most salient such question is that addressed by Hogan (1994) and to some extent by Domjan (1994): How do behavior systems develop? As Hogan points out, this question really consists of several questions. Among them are how the individual perceptual, central, and motor units composing a behavior system get organized in the first place and how they become connected to one another. Domjan's study of courtship in quail suggests that the normal sequence of male courtship and its direction toward female quail may be built up through conditioning processes like those captured experimentally in his laboratory. In contrast, other effects of experience on the development of behavior systems are not what would be expected from conditioning principles. For example, in order for a young chick's pecking to be influenced by its nutritional state, the chick must first experience pecking followed by swallowing (Hogan, 1994). Both Domjan's and Hogan's contributions illustrate how attempting to understand the role of experience in normal behavioral development can challenge learning principles and, in some cases, suggest novel mechanisms (see also Balsam \& Silver, 1994).

\section{Conclusions}

In conclusion, I note that the common theme of these articles is identified as an approach, not a theory. This is certainly appropriate at the present stage, when the usefulness for understanding learning of studying behavior systems is still being explored. As discussed earlier in this commentary, even how behavior systems are best described could be considered debatable (see Hogan, 1994). However, as a group, the articles raise a number of clear theoretical issues about how behavior systems are organized in general and how this organization affects and is affected by learning. It is striking how similar are some of the formulations and conclusions of the three articles specifically addressed to the role of behavior systems in a learning context (i.e., Timberlake's, Domjan's, and Fanselow's). At least a partial theory of behavior systems is implicit in the symposium as a whole. People working with the behavior systems approach might consider whether the time is ripe for it to yield up a more explicit theory (or theories) of behavior systems.

Finally, although the accompanying articles may have persuaded some readers that understanding a species' behavior systems may illuminate what and how its members learn, many may be unenthusiastic about incorporating a study of behavior systems into their own research. After all, a considerable amount of useful literature is published every year in which stimulus, response, and subject are almost complete abstractions, except for their specification in Method sections. Moreover, learning psychologists are not typically trained as ethologists and are often more interested in specific theoretical issues than in animal behavior per se. Painstaking observation of why and when subjects do what they do may seem unappealing or downright daunting. In either case, it may appear too much like botanizing to be theoretically satisfying. Such readers should be reminded of Timberlake's (1994) point that the behavior systems approach is complementary to, not in conflict with, other approaches to learning. The present collection of articles indicates that it is an approach that may be valuable, even indispensable, for answering some questions that arise in research using other approaches. 


\section{REFERENCES}

Baerends, G. P. (1970). The herring gull and its egg: Part C. A model of the functional organization of incubation behavior. Behaviour, 17, 263-312.

Baerends, G. P. (1976). The functional organization of behaviour. $A n$ imal Behaviour, 24, 726-738.

Balsam, P. D., \& Silver, R. (1994). Behavioral change as a result of experience: Toward principles of learning and development. In J. A. Hogan \& J. J. Bolhuis (Eds.), Causal mechanisms of behavioural development (pp. 327-357). Cambridge: Cambridge University Press.

Bolles, R. C. (1970). Species-specific defense reactions and avoidance learning. Psychological Review, 77, 32-48.

Breland, K., \& Breland, M. (1961). The misbehavior of organisms. American Psychologist, 16, 681-684.

DomJan, M. (1983). Biological constraints on instrumental and classical conditioning: Implications for general process theory. Psychology of Learning \& Motivation, 17, 215-277.

Domuan, M. (1994). Formulation of a behavior system for sexual conditioning. Psychonomic Bulletin \& Review, 1, 421-428.

FANSELOW, M. S. (1994). Neural organization of the defensive behavior system responsible for fear. Psychonomic Bulletin \& Review, 1 , $429-438$.
Gallistel, C. R. (1980). The organization of action: A new synthesis. Hillsdale, NJ: Erlbaum.

Hogan, J. A. (1994). Structure and development of behavior systems. Psychonomic Bulletin \& Review, 1, 439-450.

KONORSK1, J. (1967). Integrative activity of the brain. Chicago: University of Chicago Press.

MCFARLAND, D., \& Bösser, T. (1993). Intelligent behavior in animals and robots. Cambridge, MA: MIT Press.

RESCORlA, R. A. (1988). Pavlovian conditioning: It's not what you think it is. American Psychologist, 43, 151-160.

SHETtLEwORTH, S. J. (1975). Reinforcement and the organization of behavior in golden hamsters: Hunger, environment and food reinforcement. Journal of Experimental Psychology: Animal Behavior Processes, 104, 56-87.

SHETTLEWORTH, S. J. (1993). Varieties of learning and memory in animals. Journal of Experimental Psychology: Animal Behavior Processes, 19, 5-14.

Timberlake, W. (1994). Behavior systems, associationism, and Pavlovian conditioning. Psychonomic Bulletin \& Review, 1, 405420 .

(Manuscript received August 17, 1994; accepted for publication August 18, 1994.) 